\title{
PSA screening: Back to the future
}

M Y UROLOGIC CAREER began in the late 1980s, just before prostate-specific antigen (PSA) testing was introduced. Ever since, a busy prostate cancer practice has given me a frontline view of the benefits and possible harms of PSA screening.

See related article, page 871

In the pre-PSA era, about half of men with newly diagnosed prostate cancer presented with incurable disease, either locally advanced or metastatic. The most common treatment was bilateral orchiectomy, which was the only safe form of androgen deprivation available.

Fast-forward a few years to the mid-1990s. Within 5 years after the introduction of PSA testing, the rate of incurable disease at diagnosis fell to just 5\%, and treatment for localized disease skyrocketed, including radical prostatectomy, external beam radiation, and brachytherapy. As a result of earlier diagnosis and improved treatments, the death rate from prostate cancer in US men has fallen more than 30\% since 1990.

The first-hand experience of seeing this massive stage migration to curable disease has forever convinced me that PSA screening is beneficial. Robust statistical models lend credence to this belief, with estimates that screening is responsible for $45 \%$ to $70 \%$ of this decline in mortality. ${ }^{1}$

Fast-forward again to 2012, when the US Preventive Services Task Force (USPSTF) published a strong recommendation against screening. The recommendation had so much force that as recently as 2014 , only $11 \%$ of men at highest risk of prostate cancer in the Cleveland Clinic system were screened for it, ${ }^{2}$ mirroring national trends.

doi:10.3949/ccjm.85a.18102
What happened? Colored by the experience in the era before PSA, when men presented frequently with painful metastatic disease and had an average life expectancy of 18 to 24 months, it was widely believed that all detected prostate cancer required treatment. What was not appreciated was that while PSA detects lots of prostate cancer, the most common reason for PSA levels to reach a range worrisome enough to trigger biopsy was actually benign prostatic hypertrophy.

The resulting increase in the number of biopsies resulted in the detection of a substantial number of low-grade cancers that were never destined to cause clinical harm but that got treated anyway, based on the fear that all cancers had metastatic potential. The USPSTF based its recommendation against screening on the harms caused by this overdetection and overtreatment of nonlethal disease, focusing on risks of biopsy such as sepsis, and on treatment-related adverse effects such as changes in urinary, bowel, and sexual function.

\section{RANDOMIZED TRIALS SHOW A BENEFIT FROM SCREENING}

As a result of this controversy, several large randomized trials designed to test whether PSA screening was beneficial were organized and begun in the 1990s, with one in the United States and another in Europe., ${ }^{3,4}$ Mature data from both trials have now established that there is indeed benefit to populationlevel screening.

The US Prostate, Lung, Colorectal, and Ovarian Cancer Screening Trial (PLCO), was initially reported to show no difference in prostate cancer-specific mortality rates in those screened vs not screened, but because

\section{Screening} for prostate

\section{cancer} reduces rates of metastasis and death, and continues to evolve 
more than $90 \%$ of the men in the no-screening arm were screened anyway, that conclusion is erroneous. ${ }^{3}$

With 13-year follow-up and far less PSA contamination in the unscreened arm, the European Randomized Study of Screening for Prostate Cancer (ERSPC) in men ages 55 to 69 demonstrated a $27 \%$ reduction in the rate of death and a $35 \%$ reduction in the need for palliative treatments (androgen deprivation or radiation, or both) for metastatic disease in those screened vs not screened, clearly establishing substantial clinical benefit to PSA screening. ${ }^{4}$

A recent analysis of both trials that controlled for PSA drop-ins (comparing those actually screened with those actually not screened) concluded that the benefit of screening in terms of mortality reduction (estimated at about $30 \%$ ) are equal in both trials. ${ }^{5} \mathrm{~A}$ large cohort study from Kaiser Permanente with 16year follow-up has suggested that PSA screening has both a prostate cancer-specific benefit and an overall mortality benefit. ${ }^{6}$

\section{ACTIVE SURVEILLANCE CAN REDUCE OVERTREATMENT}

A strategy

of active

surveillance

reduces harms

of overtreating

and prevents

unnecessary

treatment

in many patients
In parallel with the design and completion of these trials, there was a significant effort to better identify and manage patients initially overdiagnosed with nonlethal cancers by developing active surveillance regimens.

This management strategy recognizes that most low-grade cancers pose no short-term risk to the patient's health or longevity, that definitive therapy can be deferred, and that with regular monitoring by digital rectal examination, PSA measurement, and repeat biopsy, cancers that progress can still be cured. The result of this strategy is a marked reduction in the harms caused by overtreatment (ie, the aforementioned adverse effects), as well as the avoidance of unnecessary treatment in many patients.

A randomized trial and 2 large prospective cohort studies have confirmed the long-term safety of this approach, ${ }^{7-9}$ and the development of commercially available, biopsy-based gene expression profiling tools promises to further improve risk stratification at diagnosis and during follow-up for individual patients. ${ }^{10}$
NEW USPSTF RECOMMENDATIONS: AN INDIVIDUAL, INFORMED DECISION

Based on the results of the ERSPC and the widespread adoption and safety of active surveillance, which together show benefit to screening and fewer harms in overdetection and overtreatment, in 2018 the USPSTF recast its recommendations. In upgrading the recommendation from "D" to "C," the recommendation now states that for men ages 55 to 69 , PSA screening should be an individual decision after a discussion with an informed provider, although men over 70 are still advised not to undergo screening at all. ${ }^{11}$

Some may think that this recommendation has arrived just in time, or that it should be made even stronger to actually recommend screening, as recent data from 2 national registries - the Surveillance, Epidemiology, and End Results program and the National Cancer Database-show that the fall in screening after the 2012 USPSTF guidelines has resulted in an increase in men presenting with advanced stage disease. ${ }^{12,13}$ (All of you Back to the Future fans, please return to the mid to late 1980s to see how that plays out.)

So the pendulum has now swung back in favor of screening, largely supported by solid data showing meaningful clinical benefit, better understanding of PSA and prostate cancer biology, and adoption of active surveillance.

\section{AN IDEAL SCREENING PROGRAM}

An ideal screening program would detect only biologically significant cancers, thus eliminating overdetection and overtreatment. There is reason for optimism on this front.

Second-generation PSA tests have better diagnostic accuracy for high-grade disease than earlier tests. Two such tests, the Prostate Health Index (Beckman Coulter) and the 4Kscore (Opko Health), are commercially available though not usually covered by commercial insurers. ${ }^{14} \mathrm{~A}$ third test, IsoPSA (Cleveland Diagnostics), is under development. Most hospital laboratories will be able to be run this test with no need for a central laboratory. ${ }^{15}$ All 3 tests have been shown to reduce unnecessary biopsies (because of a low probability of finding a biologically significant cancer) by $30 \%$ to $45 \%$ and will help reduce overdetection. 
Moreover, multiparametric magnetic resonance imaging of the prostate has been shown to improve detection of high-grade cancers, ${ }^{16}$ and a randomized trial has suggested that its incorporation into a screening strategy is costeffective and could be better than PSA testing plus transrectal ultrasonography alone (the current standard of care). ${ }^{17}$

Several risk scores based on germline genomics also hold promise for better identi-

\section{REFERENCES}

1. Etzioni R, Tsodikov A, Mariotto A, et al. Quantifying the role of PSA screening in the US prostate cancer mortality decline. Cancer Causes Control 2008; 19(2):175-181. doi:10.1007/s10552-007-9083-8

2. Misra-Hebert AD, Hu B, Klein EA, et al. Prostate cancer screening practices in a large, integrated health system: 2007-2014. BJU Int 2017; 120(2):257-264. doi:10.1111/bju.13793

3. Shoag JE, Mittal S, Hu JC. Reevaluating PSA testing rates in the PLCO trial. N Engl J Med 2016; 374(18):1795-1796. doi:10.1056/NEJMc1515131

4. Schröder FH, Hugosson J, Roobol MJ, et al; ERSPC Investigators. Screening and prostate cancer mortality: results of the European randomised study of screening for prostate cancer (ERSPC) at 13 years of follow-up. Lancet 2014; 384(9959):2027-2035. doi:10.1016/S0140-6736(14)60525-0

5. Tsodikov A, Gulati R, Heijnsdijk EAM, et al. Reconciling the effects of screening on prostate cancer mortality in the ERSPC and PLCO trials. Ann Intern Med 2017; 167(7):449-455. doi:10.7326/M16-2586

6. Alpert PF. New evidence for the benefit of prostate-specific antigen screening: data from 400,887 Kaiser Permanente patients. Urology 2018; 118:119-126. doi:10.1016/j.urology.2018.02.049

7. Lane JA, Donovan JL, Davis M, et al; ProtecT Study Group. Active monitoring, radical prostatectomy, or radiotherapy for localised prostate cancer: study design and diagnostic and baseline results of the ProtecT randomised phase 3 trial. Lancet Oncol 2014; 15(10):1109-1118. doi:10.1016/S1470-2045(14)70361-4

8. Tosoian JJ, Mamawala M, Epstein Jl, et al. Intermediate and longerterm outcomes from a prospective active-surveillance program for favorable-risk prostate cancer. J Clin Oncol 2015; 33(30):3379-3385. doi:10.1200/JCO.2015.62.5764

9. Klotz L, Vesprini D, Sethukavalan P, et al. Long-term follow-up of a large active surveillance cohort of patients with prostate cancer. J Clin Oncol 2015; 33(3):272-277. doi:10.1200/JCO.2014.55.1192

10. Nyame YA, Grimberg DC, Greene DJ, et al. Genomic scores are independent of disease volume in men with favorable risk prostate cancer: implications for choosing men for active surveillance. J Urol fying those at risk and for helping to de-intensify screening for those unlikely to have high-grade cancer. ${ }^{18}$

Screening for prostate cancer reduces mortality rates and the burden of metastatic disease, and the paradigm continues to evolve. Men at risk by virtue of age ( 55 to 69 , and healthy men $>70$ ), family history, race, and newly identified factors (germline genetics) all deserve an informed discussion on the benefits and risks of screening.
2018; 199(2):438-444. doi:10.1016/j.juro.2017.09.077

11. US Preventive Services Task Force. Final recommendation statement. Prostate cancer: screening. www.uspreventiveservicestaskforce.org/Page/Document/RecommendationStatementFinal/ prostate-cancer-screening1. Accessed October 2, 2018.

12. Negoita S, Feuer EJ, Mariotto A, et al. Annual report to the nation on the status of cancer, part II: recent changes in prostate cancer trends and disease characteristics. Cancer 2018; 124(13):2801-2814. doi:10.1002/cncr.31549

13. Weiner AB, Matulewicz RS, Eggener SE, Schaeffer EM. Increasing incidence of metastatic prostate cancer in the United States (2004-2013). Prostate Cancer Prostatic Dis 2016; 19(4):395-397. doi:10.1038/pcan.2016.30

14. Loeb S. Biomarkers for prostate biopsy and risk stratification of newly diagnosed prostate cancer patients. Urol Pract 2017; 4(4):315321. doi:10.1016/j.urpr.2016.08.001

15. Klein EA, Chait A, Hafron JM, et al. The single-parameter, structurebased IsoPSA assay demonstrates improved diagnostic accuracy for detection of any prostate cancer and high-grade prostate cancer compared to a concentration-based assay of total prostatespecific antigen: a preliminary report. Eur Urol 2017; 72(6):942-949. doi:10.1016/j.eururo.2017.03.025

16. Siddiqui MM, Rais-Bahrami S, Turkbey B, et al. Comparison of MR/ ultrasound fusion-guided biopsy with ultrasound-guided biopsy for the diagnosis of prostate cancer. JAMA 2015; 313(4):390-397. doi:10.1001/jama.2014.17942

17. Kasivisvanathan V, Rannikko AS, Borghi M, et al; PRECISION Study Group Collaborators. MRI-targeted or standard biopsy for prostate-cancer diagnosis. N Engl J Med 2018; 378(19):1767-1777. doi:10.1056/NEJMoa1801993

18. Seibert TM, Fan CC, Wang Y, et al. PRACTICAL Consortium. Polygenic hazard score to guide screening for aggressive prostate cancer: development and validation in large scale cohorts. BMJ 2018; 360:j5757. doi:10.1136/bmj.j5757

ADDRESS: Eric Klein, MD, Department of Urology, Q10-1, Glickman Urological and Kidney Institute, Cleveland Clinic, 9500 Euclid Avenue, Cleveland, $\mathrm{OH}$ 44195; kleine@ccf.org 